\title{
Taxonomic and nomenclatural notes on the genera Themus Motschulsky and Lycocerus Gorham (Coleoptera, Cantharidae)
}

\author{
Yuxia Yang', Andreas Kopetz², Xingke Yang ${ }^{3}$ \\ I College of Life Sciences, Hebei University, Baoding 071002, Hebei Province, China 2 Im Semmichbache 14, \\ D-99334 Eischleben, Germany 3 Key Laboratory of Zoological Systematics and Evolution, Institute of Zoology, \\ Chinese Academy of Sciences, Beijing 100101, China
}

Corresponding author: Yuxia Yang (yangxk@ioz.ac.cn)

Academic editor: Lyubomir Penev | Received 5 May 2013 | Accepted 26 September 2013 | Published 4 October 2013

Citation: Yang Y, Kopetz A, Yang X (2013) Taxonomic and nomenclatural notes on the genera Themus Motschulsky and Lycocerus Gorham (Coleoptera, Cantharidae). ZooKeys 340: 1-19. doi: 10.3897/zookeys.340.5470

\begin{abstract}
The following taxonomic or nomenclatural changes are proposed: Themus (s.str.) regalis (Gorham, 1889) nom. rest.; T. (s.str.) scutulatus Wittmer, 1983 = T. (s.str.) hmong Kazantsev, 2007, syn. n.; T. (Telephorops) coelestis (Gorham, 1889) = T. violetipennis Wang \& Yang, 1992, syn. n.; T. (Telephorops) uniformis Wittmer, 1983, stat. n. $=$ T. (Telephorops) cribripennis Wittmer, 1983, syn. n.; T. (Haplothemus) licenti Pic, 1938, stat. rev., resurrected from synonymy with T. coriaceipennis (Fairmaire, 1889); Lycocerus aenescens $($ Fairmaire, 1889) $=$ L. tcheonanus (Pic, 1922), syn. n.; L. asperipennis (Fairmaire, 1891) $=$ L. wangi (Švihla, 2004), syn. n.; L. borneoensis nom. n. for Athemellus atricolor (Wittmer, 1972); L. bilineatus (Wittmer, 1995) = L. amplus (Wittmer, 1995), syn. n.; L. fairmairei nom. n. et stat. rev. for Athemus dimidiaticrus (Fairmaire, 1889), originally in Telephorus, resurrected from synonymy with L. orientalis (Gorham, 1889); L. confossicollis (Fairmaire, 1891), comb. n. hereby transferred from Cantharis $=$ L. multiimpressus (Wittmer, 1997), syn. n.; L. inopaciceps (Pic, 1926) = Athemus (Athemellus) bimaculicollis (Švihla, 2005), syn. n.; L. nigratus nom. n. for L. nigricolor (Wittmer, 1972), originally in Podabrinus; L. plebejus (Kiesenwetter, 1874) = L. brunneonotaticeps (Pic, 1922), syn. n. = Cantharis rufonotaticeps Pic, 1921, syn. n.; L. swampingatus (Pic, 1916), comb. n., hereby transferred from Cantharis. The neotypes of Themus violetipennis Wang \& Yang, 1992 and Athemus (s.str.) maculithorax Wang \& Yang, 1992 are designated respectively.
\end{abstract}

\section{Keywords}

Coleoptera, Cantharidae, Themus, Lycocerus, synonym, homonym, new name, restoration name, new combination, new status, resurrection

Copyright Yuxia Yang et al. This is an open access article distributed under the terms of the Creative Commons Attribution License 3.0 (CC-BY), which permits unrestricted use, distribution, and reproduction in any medium, provided the original author and source are credited. 


\section{Introduction}

This study presents some further taxonomic and nomenclatural clarification in the cantharid genera Themus Motschulsky, 1857 and Lycocerus Gorham, 1889, based on the examination of type specimens. See Wittmer $(1961,1969,1972,1983 a, 1995)$ for prior taxonomic changes. The present work primarily focuses on the Chinese species.

\section{Material and methods}

The aedeagi and the abdominal sternite VIII of female were dissected under a stereoscopic microscope, cleared in $10 \% \mathrm{KOH}$ solution for several minutes, then placed in a droplet of glycerol and examined under a compound light microscope. Photographs of the type specimens were taken with a Canon 450D camera equipped with an EF $100 \mathrm{~mm} \mathrm{f} / 2.8$ USM lens. Line drawings were made with the aid of camera lucida attached to a Leica MZ12.5 stereomicroscope, and edited in the CorelDRAW 12 and Adobe Photoshop 8.0.1.

In the literature citations, the square brackets "[ ]" are used for my remarks and addenda. The type specimens are quoted verbatim, "[p]" indicated that the following data are printed and " $[\mathrm{h}]$ " that they are handwritten, and the quotation marks are used to separate data from different labels and a backslash "I" to separate data from different lines of the same label. The additional specimens are transliterated from Chinese labels, except those originally in English and cited in quotation marks.

The following collection codens are used in the text:

HBUM Hebei University Museum, Baoding, China;

IZAS Institute of Zoology, Chinese Academy of Sciences, Beijing, China;

MNHN Muséum national d'Histoire naturelle, Paris, France;

NHMB Naturhistorisches Museum Basel, Switzerland;

NMPC Narodni muzeum, Praha, Czech Republic.

\section{Taxonomic account}

Themus (s.str.) regalis (Gorham, 1889), nom. rest.

Telephorus regalis Gorham 1889: 103. [Synonymized with Themus imperialis (Gorham, 1889) by Wittmer 1983a: 215.]

Telephorus imperialis Gorham 1889: 102, t. 10, fig. 8. [Primary homonym, preoccupied by Telephorus imperialis Redtenbacher, 1867.]

Cantharis imperator Pic 1906: 81. [Replacement name for Telephorus imperialis Gorham, 1889, nec Redtenbacher 1867.]

Themus imperator: Jacobson 1911: 675.

Themus regalis: Jacobson 1911: 675. 
Themus (s.str.) imperialis: Wittmer 1983a: 215.

Distribution. China, Vietnam.

Remarks. Telephorus imperialis Gorham, 1889 is a primary homonym and preoccupied by Telephorus imperialis Redtenbacher, 1867, so the former is permanently invalid (ICZN, $4^{\text {th }}$ ed., article 57.2) and should be replaced by the next oldest available name among its synonyms (ICZN, $4^{\text {th }}$ ed., article 23.3.5), that is, Themus (s.str.) regalis (Gorham, 1889) should be restated as the valid name for this species.

\section{Themus (s.str.) scutulatus Wittmer, 1983}

Themus rufoscutus Pic 1926a: 35. [Secondary homonym, preoccupied by Themus rufoscutus (Pic, 1922), originally described in Cantharis.]

Themus (s.str.) scutulatus Wittmer 1983a: 208. [Replacement name for Themus rufoscutus Pic, 1926, nec Pic 1922.]

Themus (s.str.) hmong Kazantsev 2007: 54. [Replacement name for Themus rufoscutus Pic, 1926, nec Pic 1921 [1922].] syn. n.

\section{Distribution. Vietnam.}

Remarks. This species was originally described as Themus rufoscutus Pic, 1926 (located in Vietnam), which became a junior secondary homonym of T. rufoscutus (Pic, 1922) (located in Yunnan, China) since the taxonomic status of the latter was changed by Wittmer (1983a), so the former was replaced by $T$. (s. str.) scutulatus in the latter study. However, this nomenclature change was neglected by Kazantsev (2007), so that T. rufoscutus Pic, 1926 was replaced again by T. (s. str.) hmong. In the same work (Kazantsev and Brancucci 2007), the distribution of this species was recorded occurring in both Vietnam and China (Yunnan). Obviously, T. (s. str.) hmong is a junior objective synonym of $T$. (s.str.) scutulatus (ICZN, $4^{\text {th }}$ ed., article 72.7 ), which should be restricted to Vietnam and excluded from the Chinese fauna at the moment.

\section{Themus (Telephorops) coelestis (Gorham, 1889)}

http://species-id.net/wiki/Themus_coelestis

Telephorus coelestis Gorham 1889: 104, t. 10, fig. 7.

Themus coelestis: Jacobson 1911: 675.

Themus rugosus Pic 1929: 8. [Synonymized by Wittmer 1983a: 197.]

Themus (Telephorops) coelestis: Wittmer 1983a: 197, figs. 1, 59.

Themus violetipennis Wang and Yang 1992: 265, fig 2.

Themus (s.str.) violetipennis: Švihla 2008: 186. syn. n.

Type material examined. Telephorus coelestis: Lectotype $\lesssim$ (NHMB): without local-

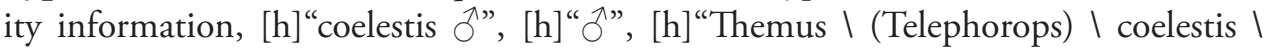


(Gorh.) \det. W. Wittmer”, [h]“Type”, [p] “LECTOTYPUS”, [p]“Naturhist. \Museum Basel \ coll. W. Wittmer", [p] "CANTHARIDAE \CANTH00001277”. Paralectotype: 1 q $(\mathrm{MNHN})$ : [p] "Kiukiang \June, $1887 \backslash$ A. E. Pratt", [h] "coelestis \Gorh.", [h] "Themus \(Telephorops) \ coelestis $\backslash$ (Gorh.) \ det. W. Wittmer", [h] "TYPE", [p] "PARALECTOTYPUS".

Themus rugosus: Holotype + (MNHN): [h] "Fokien", [h] "Themus $\backslash$ rugosus $\backslash \mathrm{n}$. sp.", [h] "Themus $\backslash$ (Telephorops) \ coelestis $\backslash$ (Gorh.) \det. W. Wittmer", [h] "type", [p]"TYPE”.

Neotype designation. Themus violetipennis: Neotype 9 (here designated, IZAS): “湖 南永顺杉木河林场 \600m \中国科学院” [Hunan, Yongshun, Shanmuhe forestry station \600m], “1988.VIII.4\采集者: 王书永” [4.VIII.1988 \leg. Shu-Yong Wang].

Additional material examined. CHINA: ShaAnXI: $2 \circ+$ (IZAS): Foping, 16.VIII.2007, leg. Yu-Xia Yang. Gansu: $1 \hat{\jmath}$ (IZAS): Kangxian, Baiyunshan, 12501750m, 12.VII.1998, leg. Shu-Yong Wang; 19 (IZAS): Kangxian, Heimaguan, 1450-1550m, 13.VII.1998, leg. De-Cheng Yuan. Henan: $1 \widehat{\jmath}$ (IZAS): Jigongshan, 700m, 14.VII.2001, leg. Si-Qin Ge; 1 ㅇ (IZAS): Tongbaishan, 500m, 16.VII.2001, leg. Si-Qin Ge; 1 (IZAS): Neixiang, Baoyunman, 21.VII.2001, leg. Fu-Qiang Chen. ANHuI: $2 \hat{\jmath}$ (NHMB): "Dabieshan, $65 \mathrm{~km}$ SW Huoshan, 1400m, 21.-24. VI.1995, leg. Bolm”. ZhejIAng: 1 ㅇ (IZAS): Xitianmushan, 23.VI.1998, leg. MingShui Zhao. Huber: $1 \hat{\jmath}, 19$ (NHMB): "Hupeh, Lichuan Distr., Suisapa, 1000m, 22.VII.1948, Gressitt \& Djou Collra”; 1 (NHMB): same data, 24.VII.1948; 1 ภ (NHMB): same data, 29.VII.1948; 20ิ $\widehat{0}, 1$ ㅇ (NHMB): same data, 31.VII.1948;

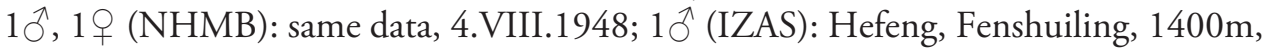
1.VIII.1981, leg. Long-Long Yang; 19 (IZAS): Hefeng, Shayuan, 30.VII.1989, leg.

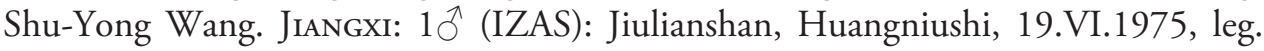
You-Wei Zhang; 1 ( (IZAS): Longnan, Jiulianshan, 17.VI.1975, leg. You-Wei Zhang. HunAN: 10, 19 (NHMB): "Yon-ping, 12.VI.1917"; 10 (NHMB): same data, "Yonping, 14.VI.1917”; 10 (IZAS): Sangzhi, Tianpingshan, 700-1450m, 14.VIII.1988,

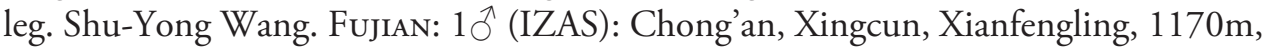
14.VII.1960, leg. Cheng-Lin Ma; 1 ( (IZAS): Dehua, Chengguan, 510-550m, 1.VI.1960, leg. Fu-Ji Pu; 11 spec. (NHMB): "Fukien, Kuatun, 15.VIII.1946, Tschung-Sen leg."; 5 spec. (NHMB): "Kuatun, 26.VII.1946"; 6 spec. (NHMB): same data, 11.VII.1946; 5 spec. (NHMB): same data, 16.VIII.1946; 4 spec. (NHMB): same data, 18.IX.1946. Hainan: 1 (ㅇ (IZAS): Wanning, 10m, 9.VI.1960, leg. ChangQing Li. GuAngXI: 1 $\overbrace{}^{\lambda}$ (IZAS): Longsheng, Tianpingshan, 740m, 17.VI.1963, leg. Shu-Yong Wang; 1 ㅇ (IZAS): Maoershan, Tongmujiang, 800m, 15.VII.1985, leg. Su-Bai Liao. Sichuan: 1ð, 1 우 (IZAS): Youyang, 780m, 15.VII.1989, leg. Shu-Yong

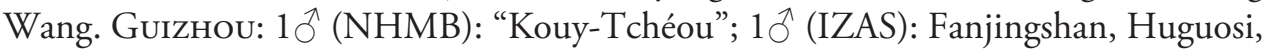
$1350 \mathrm{~m}, 3$. VIII.2001, leg. Qiong-Zhang Song; 1 ㅇ (IZAS): Fanjingshan, Huixiangping, 1600m, 2.VIII.2001, leg. Kang-Zhen Dong.

Distribution. China (Shaanxi, Gansu, Henan, Anhui, Zhejiang, Hubei, Jiangxi, Hunan, Fujian, Hainan, Guangxi, Sichuan, Guizhou). 
Remarks. According to the original publication, the types of Themus violetipennis Wang \& Yang, 1992 were deposited in the IZAS and China Agriculture University, Beijing, China (CAUB), but our search of the types in the two Chinese museums have been long, repeated and with no results. The original description of T. violetipennis was in accord with the standard of that time but insufficient considering the present level, and neotype allows us to satisfy a better comparision. Fortunately, a female specimen, which was collected at the same locality and date as that of one paratype designated by Wang and Yang (1992), was found in IZAS during our study. Its morphological characters are consistent with the original description, so it is designated as the neotype here, in order to clarify the taxonomic status of this species (ICZN, $4^{\text {th }}$, article 75.3). Furthermore, a careful examination of the types shows that Themus violetipennis Wang \& Yang, 1992 is a junior synonym of T. (Telephorops) coelestis (Gorham, 1889), which is widely distributed in China based on the data from a large series of examined specimens.

\section{Themus (Telephorops) uniformis Wittmer, 1983, stat. $\mathrm{n}$.}

Figs $1-2$

Themus (s.str.) bitinctus uniformis Wittmer 1983a: 218, fig. 30.

Themus (s.str.) cribripennis Wittmer 1983b: 151, figs. 46, 49.

Themus (Telephorops) bitinctus uniformis: Švihla 2008: 187.

Themus (Telephorops) cribripennis: Švihla 2008: 187. syn. n.

Type material examined. Themus (s.str.) bitinctus uniformis: Holotype $\hat{\sigma}$ (NHMB): [p]"Yen-ping, China \VII.21. $1917 \backslash$ Ac. 5148", [h]"bitinctus \ uniformis", [p] "HOLOTYPUS", [p] "Naturhist. \ Museum Basel \ coll. W. Wittmer", [p]"CANTHARIDAE \CANTH00000449".

Themus (s.str.) cribripennis: Holotype ${ }^{\lambda}(\mathrm{MNHN})$ : [p] "Taihorinsho \Formosa \Sauter, VIII_7_09", [h] "Cantharis \ davidis Fairm.", [h] "Themus (s.str.) \ cribripennis \Wittm. \ det. W. Wittmer”, [p]"HOLOTYPUS”, [h] “136”. Paratype: 1 우 (NHMB): [p]"Suisharyo \Formosa \H. Sauter, X.1911", [h] "Themus s.str. \ cribripennis \Wittm. \ det. W. Wittmer", [p] "PARATYPUS”, [p] "CANTHARIDAE \ CANTH00002654".

Distribution. China (Fujian, Taiwan).

Remarks. Having examined the holotypes of Themus cribripennis Wittmer, 1983b and T. bitinctus uniformis Wittmer, 1983a, we were unable to find differences justifying their separation, which has led us to consider all the examined specimens of both nominal species to be conspecific. Therefore, we synonymized $T$. cribripennis under $T$. bitinctus uniformis. Furthermore, T. bitinctus uniformis should be upgraded to the specific rank, because it is obviously different from T. bitinctus Wittmer, 1982 (located in Vietnam) in the aedeagus, except the difference in the elytra coloration from the latter. 

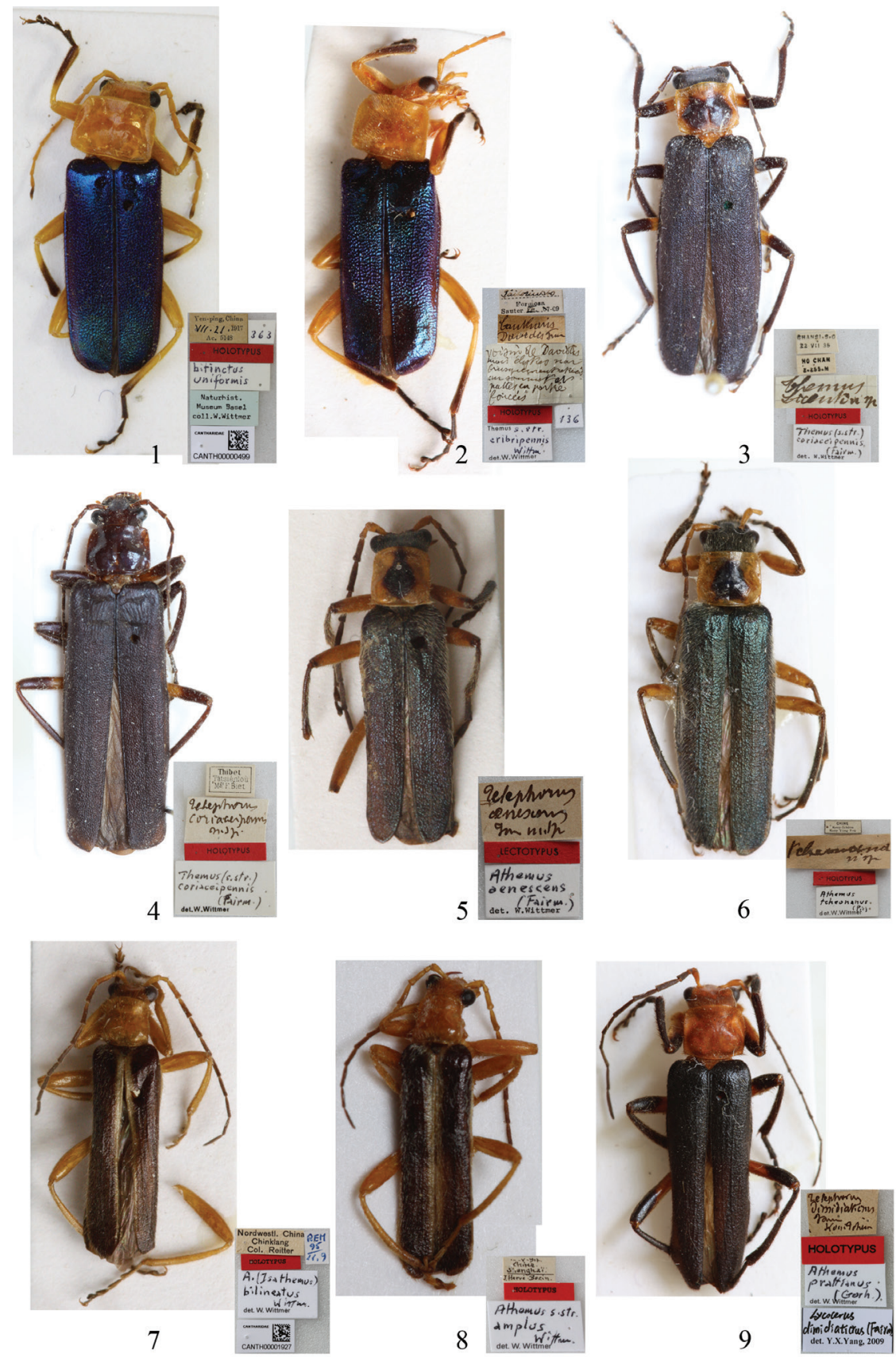

Figures I-9. Habitus, dorsal view I Holotype of Themus (s.str.) bitinctus uniformis Wittmer, 1983 2 Holotype of T. (s.str.) cribripennis Wittmer, 19833 Holotype of T. licenti Pic, 19384 Holotype of Telephorus coriaceipennis Fairmaire, 18895 Lectotype of Podabrus aenescens Fairmaire, 18896 Holotype of Cantharis tcheonana Pic, 19227 Holotype of Athemus (Isathemus) bilineatus Wittmer, 19958 Holotype of Athemus (s.str.) amplus Wittmer, 19959 Holotype of Telephorus dimidiaticrus Fairmaire, 1889. 1-2, 5, 7-8, 9 male 3-4, 6 female. 


\section{Themus (Haplothemus) licenti Pic, 1938, stat. rev.}

Fig. 3

Themus licenti Pic 1938a: 161. [Synonymized with Themus coriaceipennis (Fairmaire, 1889) by Wittmer 1983a: 224.]

Themus coriaceipennis (Fairmaire, 1889): Wittmer 1983a: 224, figs 43, 111. [Misidentification.]

Type material examined. Themus licenti: Holotype $q$ (MNHN): [p]"CHANSI. S.O. $\backslash$ 22.VII.35", [p] "HO CHAN \2,255m", [h] "Themus \licenti n. sp.", [h] "Themus (s.str.) \coriaceipennis $\backslash$ (Fairm.) $\backslash$ det. W. Wittmer", [p]"HOLOTYPUS”.

Telephorus coriaceipennis: Holotype $q$ (MNHN): [p] "Thibet $\backslash$ Tàtsiénloù $\backslash \mathrm{M}$. F. Biet", [h] “Telephorus $\backslash$ coriaceipennis $\backslash$ n. sp.”, [h] “Themus (s.str.) \coriaceipennis $\backslash$ (Fairm.) \det. W. Wittmer", [p] "HOLOTYPUS”.

Additional material examined. CHINA: HenAN: $1 \hat{\gamma}, 1 q$ (IZAS): Lushi, Jihe forestry station, 1200m, 20.VII.2001, leg. Kang-Zhen Dong. Sichuan: $50^{\lambda}{ }^{\lambda}, 1 q$ (NHMB): "Szechuen, Yao Gi, nr Mupin, 7400ft., 15.VII.1929, D. C. Graham"; 1ठ, 1 ( NHMB): "Mu San Tsai, 10km NW Weichow, 8700ft., 26.-28.VI.1933, D. C. Graham"; 10̄, 1 q (IZAS): Luding, Xinxing, Yanzigou, 1560m, 7.VIII.2004, leg. Ming Bai.

Distribution. China (Henan, Shaanxi, Sichuan).

Remarks. Themus licenti Pic, 1938a was synonymized with T. coriaceipennis (Fairmaire, 1889) by Wittmer (1983a). However, examination of the holotypes of both nominal species shows that they are different species. Themus licenti is obviously different from T. coriaceipennis in the following characters: head (Fig. 3) width across eyes wider than anterior margin of pronotum, pronotum reddish brown with a large black marking in middle, abdominal sternite VIII of female (see Wittmer 1983a: fig. 111) deeply concaved on both sides of the middle emargination of posterior margin; while in T. coriaceipennis (Fig. 4), head width across eyes narrower than anterior margin of pronotum, pronotum uniformly dark brown, without any black marking, abdominal sternite VIII of female (Fig. 22) slightly concaved on both sides of the middle emargination of posterior margin. Therefore, we suggest Themus licenti Pic, 1938a be resurrected from synonymy with $T$. coriaceipennis (Fairmaire, 1889).

\section{Lycocerus aenescens (Fairmaire, 1889)}

http://species-id.net/wiki/Lycocerus_aenescens

Figs 5-6

Podabrus aenescens Fairmaire 1889: 40.

Cantharis tcheonana Pic 1922: 31.

Themus angusticollis Pic 1955: 16. [Secondary homonym, preoccupied by Athemus angusticollis (Gorham, 1882), originally described in Telephorus. Synonymized with Athemus tcheonanus (Pic, 1922) by Wittmer 1995: 211.] 
Athemus angustithorax Wittmer 1975: 260. [Replacement name for Themus angusticollis Pic, 1955, nec Gorham 1882.]

Athemus tcheonanus: Wittmer 1975: 260; 1995: 211, figs. 42, 43, 168, 169.

Athemus aenescens: Wittmer 1982: 341.

Lycocerus aenescens: Kazantsev and Brancucci 2007: 249.

Lycocerus tcheonanus: Kazantsev and Brancucci 2007: 254. syn. n.

Type material examined. Podabrus aenescens: Lectotype $\widehat{\partial}(\mathrm{MNHN})$ : without locality information, [h] "Telephorus aenescens $\backslash$ Frm., n. sp.", [h] "Athemus $\backslash$ aenescens $\backslash$ (Fairm.) \ det. W. Wittmer", [p] “LECTOTYPUS”. Paralectotype: 1 ㅇ (MNHN): [p] "Kouy-Tchèu", [p]"PARALECTOTYPUS".

Cantharis tcheonana: Holotype $q$ (MNHN): [p]"CHINE \Kouy-Tchèou \Koúy Yang Foú", [h] "tcheonana \n. sp.", [h] "Athemus \tcheonanus $\backslash$ (Pic) $\backslash$ det. W. Wittmer", [p] "HOLOTYPUS".

Themus angusticollis: Holotype $\widehat{\partial}(\mathrm{MNHN})$ : [p] "Chasseurs Indigenes \des Missionnaires $\backslash$ de Ta-tslen-Lou $\backslash 1906$ ", [h] "Themus $\backslash$ angusticollis $\backslash$ n. sp.", [h] "Athemus s.str. \angustithorax \Wittm. \det. W. Wittmer”, [h] "Athemus s.str. \ tcheonanus \ (Pic) \det. W. Wittmer", [p]“HOLOTYPUS".

Additional material examined. CHINA: GuANGXI: 10 , 1 ㅇ (NHMB): "KoungSi-Hien, alt. 2100m". Sichuan: $1 \overbrace{}^{\Uparrow}$ (NHMB): "Moxi, Gongashan mts., 1650m, 28.VI-2.VII.1994, leg. Bolm”. Guizhou: 10 (MNHN): "Kouy-Tchèu, Koúy Yang Foú"; 2 우 (MNHN): "Kouy-Tchèu"; 1 ( $(\mathrm{MNHN}):$ "Kouei-Tcheou"; 5 우 (MNHN): "Kuoy-Tcheou, Gan chouem, Min y fou et Tchen-Fong, Tchéou, 1918, P. Cavalerie"; 1 + (MNHN): "Kouy-Tchèu, 1921, Cavaleri"; 19 (MNHN): "KouyTchèu, Reg. De Pin-fa, 1908, Père Cavaleri”. Yunnan: $10^{\Uparrow}$ (MNHN): “Tse Kou, 1895, R. P. Dubernard”; 10๊, 1 ㅇ (IZAS): Dali, Cangshan, 30.V.1955, leg. Xing-Chi Yang; 8 spec. (NHMB): "Cangshan mts., E slope, 2000-2500m, 25 42 'N, 100 $08^{\prime} \mathrm{E}$, 21.VI.1992, leg. Vit Kubáń”; 12 spec. (NHMB): "Dali, 19.-21.V.1993, leg. R. Cervenka”; $2 \widehat{\jmath}, 1$ ㅇ (NHMB): "Dali, 1600-2000m, 5.-8.VII.1990, leg. L. M. Bocák"; 10̂, 2 우 (NHMB): "Dali, 1.-7.VI.1994, B. Šiška et T. Spevár”.

Distribution. China (Guangxi, Sichaun, Guizhou, Yunnan).

Remarks. Having examined the holotypes of Lycocerus aenescens (Fairmaire, 1889) and L. tcheonanus (Pic, 1922), we could not find any difference to justify their separation, so we synonymize $L$. tcheonanus under $L$. aenescens.

\section{Lycocerus asperipennis (Fairmaire, 1891)}

http://species-id.net/wiki/Lycocerus_asperipennis

Telephorus asperipennis Fairmaire 1891: ccviii.

Cantharis limbatipennis Pic 1906: 83. [Synonymized by Wittmer 1995: 256.]

Cantharis asperipennis: Jacobson 1911: 679.

Cantharis stötzneri Pic 1926b: 154. [Synonymized by Wittmer 1995: 256.] 
Athemus stötzneri: Pic 1933: 4.

Athemus limbatipennis: Wittmer 1972a: 106.

Athemus (s.str.) maculithorax Wang and Yang 1992: 264, fig. 1. [Secondary homonym, preoccupied by Athemus maculithorax (Wittmer, 1972), originally described in Athemellus.]

Athemus (s.str.) asperipennis: Wittmer 1995: 256, figs. 113, 114.

Athemus (s.str.) wangi Švihla 2004: 183 [Replacement name for Athemus maculithorax

Wang \& Yang, 1992, nec Wittmer 1972.]

Lycocerus asperipennis: Kazantsev and Brancucci 2007: 249.

Lycocerus wangi: Kazantsev and Brancucci 2007: 254. syn. n.

Type material examined. Telephorus asperipennis: Lectotype $\bigcirc$ (MNHN): [h] "Changyang”, [h] "Telephorus \asperipennis $\backslash$ Fairm. \Changyang”, [h] "Athemus $\backslash$ asperipennis $\backslash$ (Fairm.) \det. W. Wittmer”, [p] “LECTOTYPE”. Paralectotype: 1 ( $(\mathrm{MNHN})$ : [h] "Chang-yang”, [p] "PARALECTOTYPE”.

Cantharis limbatipennis: Lectotype + (MNHN): [h] "Yunnan $\backslash(\mathrm{China})$ ", [h] "C. limbatipennis \Pic", [h] "limbatipennis $\backslash$ Pic", [h] "von \asperipennis \Frm.", [h] "Athemus $\backslash$ asperipennis $\backslash$ (Frm.) \det. W. Wittmer", [h] “type”, [p] “TYPE”, [p] “LECTOTYPE”. Paralectotype: 1 ( (MNHN): [h] "Yunnan”, [p] "PARALECTOTYPE”.

Cantharis stötzneri: Lectotype $\widehat{\sigma}(\mathrm{MNHN})$ : [p] "Szetschwan \Kwanhsien \Exp. Stötzner”, [h] "stötzneri \n. sp.”, [h] “Athemus \asperipennis \(Frm.) \det. W. Wittmer", [p] "LECTOTYPE”. Paralectotypes: 10̄, 5 우 (MNHN): same data to lectotype, [p] "PARALECTOTYPE".

Neotype designation. Athemus maculithorax: Neotype $\widehat{\jmath}$ (here designated, IZAS): [p]“湖北兴山龙门河 \1300m” [Hubei, Xingshan, Longmenhe $\backslash 1300 \mathrm{~m}$ ], [p]“1993. VI.21 \采集者: 黄润质”[21.VI.1993 \leg. Run-Zhi Huang].

Additional material examined. CHINA: GANsu: $1 \overbrace{}^{\Uparrow}$ (IZAS): Wenxian, Qiujiaba, 2360-2650m, 29.VI.1998, leg. De-Cheng Yuan; 1 ㅇ (IZAS): same data, 30.VI.1998. Shanxi: 1 (IZAS): "Shansi, Kwashan, 9.VI.1936”. ShaAnxi: 2ðð (NHMB): "Danfeng-NE env., 900-1500m, 3345-52'N, 110²2-37'E, 28.-29.V.1995, leg. L. R. Businský"; $3 \hat{\jmath}, 1$ 을 (NHM): "Qinling Mts.-N. slpoe, Huxian Co., 1300-1600m, $33^{\circ} 50^{\prime} \mathrm{N}, 108^{\circ} 26^{\prime} \mathrm{E}, 12 .-13 . V I .1995$, leg. L. R. Businský”. Henan: 3 q $q$ (IZAS): Lushi, Jihe forestry station, 20.VII.2001, leg. Kang-Zhen Dong. Hubei: $1 \hat{\jmath}$ (NHMB): "Shennongjia, Yanzi Pass, 2200m, 31ํ3'N, 110²8'E, 23. -26.VI.1995, leg. L. R. Businský"; 1 ㅇ (NHMB): "Dashennongjia massif-E slpoe, 3124-30'N, $110^{\circ} 21-$ 24'E, 2000m, 28.VI-7.VII.1995, leg. L. R. Businský”; 1ð̄, 1 ( (IZAS): Foping, Liangfengya, 1750-2150m, 28.VI.1999, leg. YAO Jian. Sichuan: 10 spec. (NHMB): "Mt. Emei, 500-1200m, 29³0'N, 103²0'E, 4.-18.V.1989, leg. S. J. Kolibáč"; 12 spec. (NHMB): "Mt. Emei, 600-1050m, 5.-19.V.1989, leg. Lad. Bocák”; 25 spec. (NHMB): "Kwanhsien, Exp. Stötzner"; 10̄, 1 q (NHMB): "Chengtu, 1700m, 1.2.V.1933, D.C. Graham"; 1ठ̄, 1 ( NHMB): "Kuausien, 1934, D.C. Graham"; 1ð̄, 1 ㅇ (NHMB): "Yaogi nr. Mupin, 8000ft, 14.-18.VI.1929, D.C. Graham"; 1 (NHMB): "Shikaizi, Mt. Omei, 4500ft, 1945, D.C. Graham"; 1 đ (IZAS): Mt. 
Emei, Baoguosi, 550-750m, 27.IV.1957, leg. Fu-Xing Zhu; 1 ㅇ (IZAS): Mt. Emei, Qingyinge, 800-1000m, 20.IV.1957, leg. Fu-Xing Zhu; 1 ㅇ (IZAS): same locality, 30.IV.1957, leg. Ke-Ren Huang; $1{ }^{\Uparrow}$ (IZAS): same locality, 24.IV.1957, leg. Zong-Yuan Wang.

Distribution. China (Gansu, Shanxi, Shaanxi, Henan, Hubei, Sichuan, Yunnan).

Remarks. The neotype is designated for Athemus (s.str.) maculithorax Wang \& Yang, 1992 here, according to the loss of the type, and for allowing a comparision based on most of the criteria (ICZN, $4^{\text {th }}$, article 75.3). The latter's name was replaced by Athemus wangi by Švihla (2004) and now is placed in Lycocerus (Kazantsev and Brancucci 2007). Having examined the lectotype of $L$. asperipennis and a large series of additional specimens from China, we suggest $L$. wangi is a junior synonym of $L$. asperipennis, since that we could not find any difference to justify their separation.

\section{Lycocerus borneoensis Y. Yang \& X. Yang, nom. n.}

Podabrinus atricolor Pic 1938a: 158.

Pseudoabsidia atricolor: Wittmer 1969: 128.

Athemellus atricolor: Wittmer 1972a: 126 [inc. sed.]; Delkeskamp 1977: 47. [Secondary homonym, preoccupied by Lycocerus atricolor (Pic, 1922), originally described in Cantharis.]

Distribution. Borneo.

Etymology. The new name is derived from this species' type locality "Borneo".

Remarks. This species was located in Borneo and originally described in Athemellus Wittmer, 1972, which was synonymized with Lycocerus Gorham, 1889 by Okushi$\mathrm{ma}$ (2005), so it should be placed in the latter genus for the time being. Because of this change, this species and Lycocerus atricolor (Pic, 1922) (originally in Athemus) become secondary homonyms and the junior is invalid (ICZN, $4^{\text {th }}$, article 57.3.1), so its name is replaced by L. borneoensis nom. $\mathrm{n}$. here.

\section{Lycocerus bilineatus (Wittmer, 1995)}

http://species-id.net/wiki/Lycocerus_bilineatus

Figs $7-8$

Athemus (Isathemus) bilineatus Wittmer 1995: 275, figs. 140, 141.

Athemus (s.str.) amplus Wittmer 1995: 278, figs. 146, 147, 203.

Lycocerus amplus: Kazantsev and Brancucci 2007: 249. syn. n.

Lycocerus bilineatus: Kazantsev and Brancucci 2007: 249.

Type material examined. Athemus (Isathemus) bilineatus: Holotype $\delta$ (NHMB): [p]"Nordwestal. China \Chinkiang \Col. Reitter", [h] "REM \95\11.9", [h]"A. 
(Isathemus) \ bilineatus \ Wittm., \ det. W. Wittmer”, [p] “HOLOTYPUS”, [p] "CANTHARIDAE \CANTH00001927".

Athemus (s.str.) amplus: Holotype $\widehat{0}$ (MNHN): [h]"V.1917 \China \Shanghai \J. Hervé-Bdzin", [h] "Athemus s.str. \ amplus \Wittm. \ det. W. Wittmer", [p] "HOLOTYPUS”. Paratypes: $30^{\lambda}{ }^{\lambda}, 2$ 우 (MNHN): [h] “Shanghai”, [p] "PARATYPUS"; 10 (NHMB): [p] "Shanghai \V.1917 \J. Hervé-Bdzin", [p]"PARATYPUS", "CANTHARIDAE । CANTH00001340"; 1 ㅇ (NHMB): [p] "Shanghai \V $\backslash 1917$, J. Hervé-Bdzin", [p] "PARATYPUS", [p] "CANTHARIDAE \ CANTH00002072”; $1{ }^{\Uparrow}$ (NHMB): [p] “Zi-ka-wei \20.IV.1924”, [p] “PARATYPUS”, [p] "CANTHARIDAE $\backslash$ CANTH00001986".

Additional material examined. CHINA: SHANGHAI: $1{ }^{\lambda}, 2 q q$ (IZAS): "Shang-

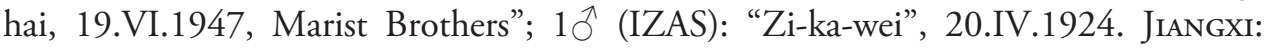

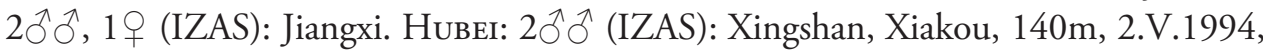
leg. Xing-Ke Yang; 2 ㅇ (IZAS): Zigui, Jiulingtou, 110m, 1.V.1994, leg. Wen-Zhu Li.

Distribution. China (Jiangsu, Shanghai, Jiangxi, Hubei).

Remarks. Both Lycocerus amplus (Wittmer, 1995) and L. bilineatus (Wittmer, 1995) were originally described in Athemus and assigned to different subgenera. In the original manuscript (Wittmer 1995), L. bilineatus was described on a single male type, so it made no sense that it was attributed to the subgenus Isathemus because of no female available. Furthermore, having examined the holotypes of both nominal species and some paratypes, as well as a series of additional specimens, we could not find any difference justifying their separation, even in the tarsal claws, which is the character to distinguish the subgenera Athemus and Isathemus (Wittmer 1995). Consequently, we synonymized $L$. amplus under $L$. bilineatus.

\section{Lycocerus fairmairei Y. Yang \& X. Yang, nom. n. et stat. rev.}

Figs 9, 19-21, 23

Telephorus dimidiaticrus Fairmaire 1889: 41.

Athemus dimidiaticrus: Wittmer 1972a: 106. [Synonymized with Athemus orientalis

(Gorham, 1889) by Wittmer 1995: 255. Secondary homonym, preoccupied by

Lycocerus dimidiaticrus (Fairmaire, 1889: 40), originally in Podabrus.]

Lycocerus orientalis (Gorham, 1889): Kazantsev and Brancucci 2007: 252.

Type material examined. Telephorus dimidiaticrus: Holotype đo (MNHN): [h] "Telephorus \dimidiaticrus \Fairm. \Koui Tchéou", [h] "Athemus \prattianus \ (Gorh.) \ det. W. Wittmer", [h] "Lycocerus $\backslash$ dimidiaticrus (Fairm.) $\backslash$ det. Y. X. Yang, 2009”, [p] "HOLOTYPUS".

Additional material examined. CHINA: FuJIAN: $10^{\lambda}$ (NHMB): "Fukien. Kua-

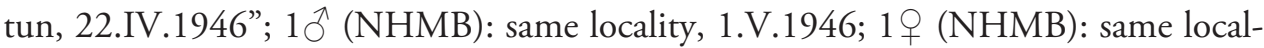
ity, 2.V.1946; 19 (IZAS): Jianyang, Huangkeng, Aotou, 950m, 5.V.1960, leg. Yong Zuo; $1 \hat{\jmath}$ (IZAS): same locality, 800-1050m, 26.IV.1960, leg. Cheng-Lin Ma. 
Distribution. China (Fujian, Guizhou).

Etymology. The new name is named after L. Fairmaire, the taxonomist who described this species.

Remarks. Fairmaire described a Podabrus dimidiaticrus Fairmaire, 1889: 40 which became Athemellus dimidiaticrus by Wittmer (1972b) and now Lycocerus dimidiaticrus (Fairmaire, 1889), which however was neglected by Kazantsev and Brancucci (2007), and in the same original publication also a Telephorus dimidiaticrus Fairmaire, 1889: 41 which became Athemus dimidiaticrus by Wittmer (1972a) and now to be placed in Lycocerus, the two species become secondary homonyms and the junior is invalid (ICZN, $4^{\text {th }}$, article 57.31.), so that a new name is needed, Lycocerus fairmairei nom. n., to replace the name of the latter species.

At the same time, having examined the holotype of Telephorus dimidiaticrus and lectotype of Lycocerus orientalis (Gorham, 1889) (10 (NHMB): [p]"Foochau \April, 1886 \Leech.”, [h] “A. orientalis", [p] “LECTOTYPE”, [p] “CANTHARIDAE । CANTH00001609”.), we found that the former is distinctly different from the latter in the aedeagus (Figs 19-21; the latter's, see Wittmer 1995: figs. 109, 110), so we suggest Telephorus dimidiaticrus Fairmaire, 1889: 41, with its new replacement name Lycocerus fairmairei nom. $\mathrm{n}$., to be resurrected from synonymy with $L$. orientalis.

\section{Lycocerus confossicollis (Fairmaire, 1891) comb. $\mathbf{n}$.}

http://species-id.net/wiki/Lycocerus_confossicollis

Figs $10-11$

Telephorus confossicollis Fairmaire 1891: ccviii.

Cantharis confossicollis: Jacobson 1911: 679.

Athemus (s.str.) multiimpressus Wittmer 1997: 285, figs. 128, 129.

Lycocerus multiimpressus: Kazantsev and Brancucci 2007: 252. syn. n.

Type material examined. Telephorus confossicollis: Lectotype $\widehat{\delta}(\mathrm{MNHN})$ : [h] "Changyang", [h] "Telephorus \confossicollis $\backslash$ Fairm. \Tchangyang", [h] "Athemellus $\backslash$ confossicollis \(Fairm.) \det. W. Wittmer”, [p] "LECTOTYPE”.

Athemus (s.str.) multiimpressus: Holotype đo (NHMB): [p]"Chin-ling Mts. । Shensi. E. B. \Apr.-May,1904”, [h] "Athemus \ multiimpressus \Wittm. \ det. W. Wittmer", [p] "HOLOTYPUS", [h] "REM \95 \1314", [p] "CANTHARIDAE । CANTH00001208".

Additional material examined. CHINA: HubEr: $1 \overbrace{}^{\lambda}$ (IZAS): Xingshan, Longmenhe, 6.V.1994, leg. Xing-Ke Yang; 1 ( (IZAS): same data, 9.V.1994; 1 q (IZAS): same locality, 9.V.1994, leg. You-Wei Zhang; $1 \overbrace{}^{\Uparrow}$ (IZAS): same locality, $730 \mathrm{~m}$, 22.VI.1994, leg. Jian Yao.

Distribution. China (Shaanxi, Hubei).

Remarks. Although the type specimen of Cantharis confossicollis was attached with Wittmer's manuscript label "Athemellus confossicollis (Fairm.)", it has never been pub- 

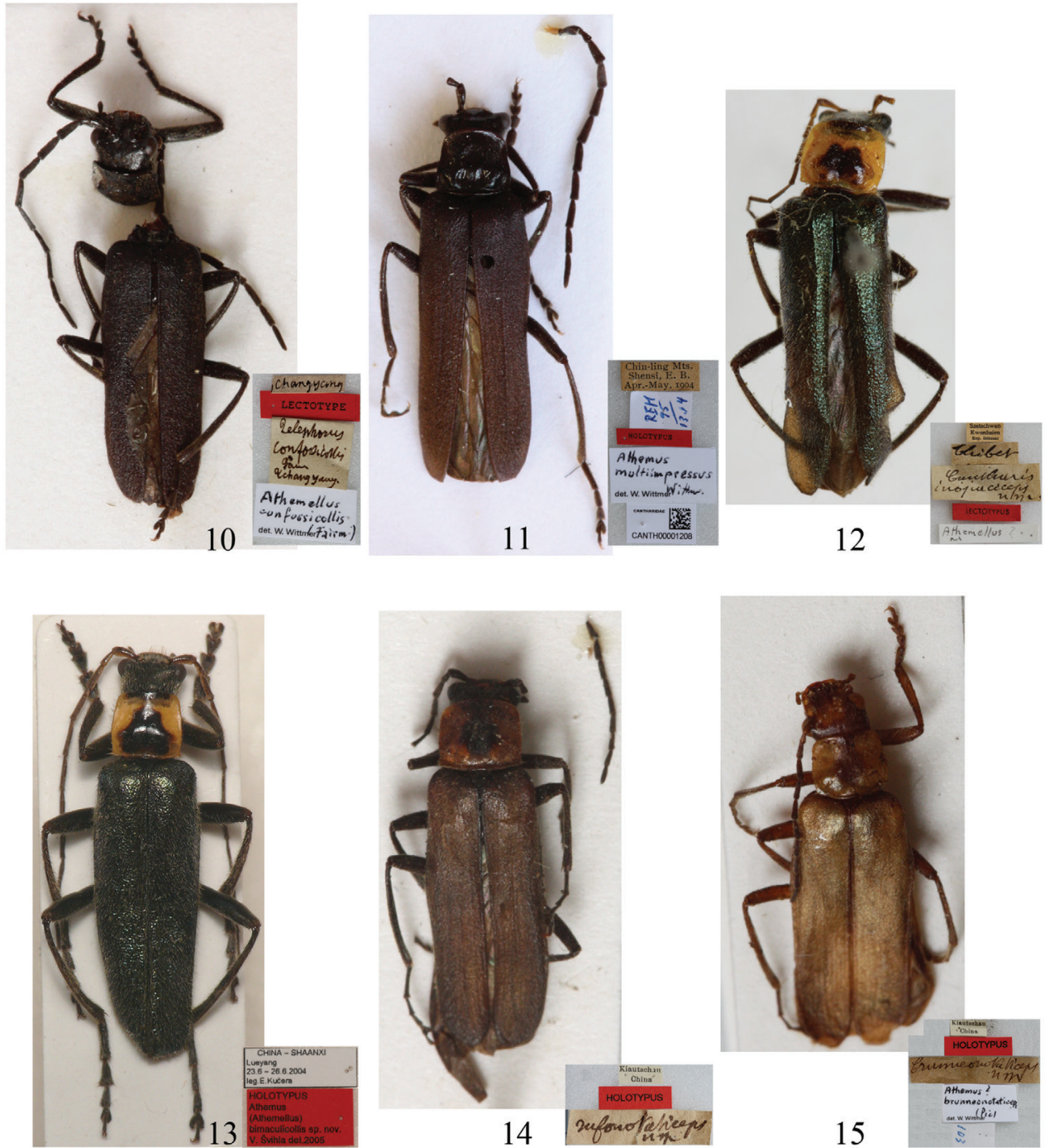

Figures I 0-15. Habitus, dorsal view I 0 Lectotype of Telephorus confossicollis Fairmaire, 1891 I I Holotype of Athemus (s.str.) multiimpressus Wittmer, 1997 I2 Lectotype of Cantharis inopaciceps Pic, 1926 13 Holotype of Athemus (Athemellus) bimaculicollis Švihla, 2005 I 4 Holotype of Cantharis rufonotaticeps Pic, 1921 I5 Holotype of $C$. brunneonotaticeps Pic, 1922. 10-11, 13 male 12, 14-15 female.

lished formally for this taxonomic change. In our opinion, this species is definitely a member of Lycocerus due to the following characters: pronotum subquadrate, all tarsal claws simple and the aedeagus with dorsal plates of parameres separated. At the same time, Lycocerus multiimpressus (Wittmer, 1997) is considered to be a junior synonym of $L$. confossicollis (Fairmaire, 1891) comb. n., since we could not find differences between both nominal species in their morphological characters, including appearance and aedeagus. Therefore, we suggest $L$. multiimpressus is a new subjective synonym of $L$. confossicollis. 
Lycocerus inopaciceps (Pic, 1926)

http://species-id.net/wiki/Lycocerus_inopaciceps

Figs $12-13$

Cantharis inopaciceps Pic 1926b: 153.

Themus inopaciceps: Wittmer 1961: 362.

Athemellus inopaciceps: Wittmer 1983a: 190.

Athemus (Athemellus) bimaculicollis Švihla 2005: 90, figs 38-41. syn. n.

Lycocerus inopaciceps : Kazantsev and Brancucci 2007: 250.

Type material examined. Cantharisinopaciceps: Lectotype $q$ (MNHN): [p] "Szetschwan $\backslash$ Kwanhsien \Exp. Stözner", [h] "Thibet”, [h] "Cantharis \inopaciceps \n. sp.”, [h] "Athemellus ?", [p] "LECTOTYPUS".

Athemus (Athemellus) bimaculicollis: Holotype $\widehat{o}$ (NMPC): [p]"CHINASHAANXI \Lueyang \23.6-26.6.2004 \leg. E. Kučera”, [p]"HOLOTYPUS \Athemus \(Athemellus) \bimaculicollis sp. nov.। V. Švihla det. 2005”. Paratype: 1 q (NMPC): same data.

Additional material examined. CHINA: SHAANXI: $1 \hat{\jmath}, 1 q$ (HBUM): Liuba, 10.-12.VI.2005, leg. Yi-Bin Ba; $1 \hat{\jmath}, 1$ ㅇ (HBUM): Liuba, Miaotaizi, 14.-15.VI.2005, leg. Yi-Bin Ba; $1 \delta^{\lambda}, 4$ 우 (HBUM): Fengxian, Heigou, 13.VI.2005, leg. Yi-Bin Ba; 3 우우 (HBUM): Liuba, Zaomulan, 11.VI.2005, leg. Yi-Bin Ba.

Distribution. China (Shaanxi, Sichuan).

Remarks. Athemus (Athemellus) bimaculicollis Švihla, 2005, which was omitted in the catalogue by Kazantsev and Brancucci (2007), is considered to be a junior synonym of Lycocerus inopaciceps (Pic, 1926), because we could not find any difference between their holotypes in the morphology including body size and coloration, tarsal claws and abdominal sternite VIII of female.

\section{Lycocerus nigratus Y. Yang \& X. Yang, nom. n.}

Podabrinus nigricolor Wittmer 1951: 99.

Pseudoabsidia nigricolor: Wittmer 1969: 128.

Athemellus nigricolor: Wittmer 1972b: 124.

Lycocerus nigricolor: Kazantsev and Brancucci 2007: 252. [Secondary homonym, preoccupied by Lycocerus nigricolor (Pic, 1938).]

Distribution. China (Fujian).

Etymology. The new specific name is derived from Latin word "niger" = black, a reference to its black body coloration, as the same meaning as its original name.

Remarks. This species was originally described in Podabrinus, its original name is a junior secondary homonym of Lycocerus nigricolor (Pic, 1938) (originally in Athemus, located in Malaya), so it was replaced by Lycocerus nigeratus nom. n. (ICZN, $4^{\text {th }}$, article 57.3.1). 


\section{Lycocerus plebejus (Kiesenwetter, 1874)}

http://species-id.net/wiki/Lycocerus_plebejus

Figs $14-15$

Cantharis plebeja Kiesenwetter 1874: 278.

Lycocerus plebejus: Okushima 2005: 114-116, figs 11e, 12g, 14e, 32, 90.

Cantharis rufonotaticeps Pic 1921: 29. syn. n.

Cantharis brunneonotaticeps Pic 1922: 32.

Athemus brunneonotaticeps: Wittmer 1995: 268, fig. 199.

Lycocerus brunneonotaticeps: Kazantsev and Brancucci 2007: 249. syn. n.

Type material examined. Cantharis rufonotaticeps: Holotype of (MNHN): [p]"Kiautschau \China", [h] "rufonotaticeps $\backslash$ n. sp.", [p] "HOLOTYPUS”.

Cantharis brunneonotaticeps: Holotype $+(\mathrm{MNHN}):[\mathrm{p}]$ "Kiautschau \China", [h] "brunneonotaticeps \ n. sp.", [h] "Athemus? \brunneonotaticeps $\backslash$ (Pic) \det. W. Wittmer", [p] "HOLOTYPUS".

Additional material examined. CHINA: ShanghaI: 10 (NHMB): "China, Kiangsu Prov., Shanghai, 15.IV.1932, A. Bavio coll."; 10, 1 ㅇ (IZAS): "China, Kiangsu Prov., Shanghai, Zi-ka-wei, 20.IV.1924". Jiangxi: 19 (MNHN): "Süd-

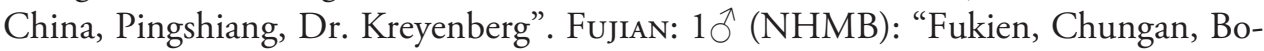
hea Hill, 15.III.1940, coll. T. C. Maa"; 1 (NHMB): same data, 16.III.1940; 10,

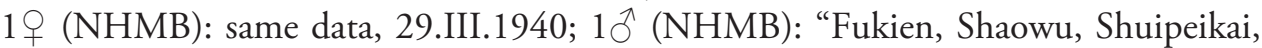
3.IV.1942, coll. T. C. Maa"; $1{ }^{\Uparrow}$ (NHMB): "Fukien, Shaowu, Kuhsienkai, IV.1944, coll. T. C. Maa"; 19 (NHMB): "Fukien, Chungan, Kuatun, IV.1942, coll. T. C. Maa”; $1 \widehat{\jmath}$ (IZAS): Shaowu, Chengguan, 150-220m, 19.IV.1960, leg. Cheng-Lin Ma; 1 (IZAS): same locality, 160-210m, 17.III.1960, leg. Yong Zuo; 19 (IZAS): same locality, 150-190m, 18.III.1960, leg. Yong Zuo. GuAngxi: $1 \hat{\sigma}^{\hat{O}}$ (IZAS): Guilin, Liangfeng, 17.III.1952; 19 (IZAS): same locality, 26.III.1952. Sichuan: 1 ㅇ (IZAS): Mt. Emei, Baoguosi, 550-750m, 7.IV.1957, leg. Ke-Ren Huang; 10 (IZAS): same locality, 12.IV.1957, leg. You-Cai Yu; 1 (IZAS): same data, 19.IV.1957; 1 (IZAS): same data, 3.IV.1957.

Distribution. China (Shandong, Shanghai, Jiangxi, Fujian, Guangxi, Sichuan).

Remarks. Having examined the holotypes of Cantharis rufonotaticeps Pic, 1921 and Lycocerus brunneonotaticeps (Pic, 1922) (originally in Cantharis), as well as a large series of additional specimens including both sexes, we were unable to find differences justifying their separation, although some variation in the coloration of head and elytra, which has led us to consider all the examined specimens of both nominal species to be conspecific. Furthermore, we discovered that their characters are consistent with the redescription and illustrations of Lycocerus plebejus (Kiesenwetter, 1874) provided by Okushima (2005). Consequently, we synonymized Cantharis rufonotaticeps and Lycocerus brunneonotaticeps under Lycocerus plebejus, which confirmed Okushima's presum that the latter occurs in China but Japan. 


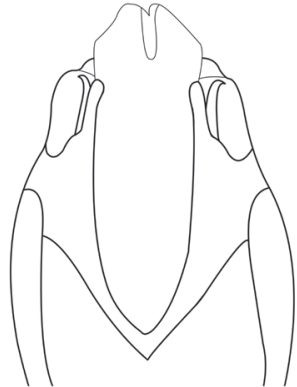

16

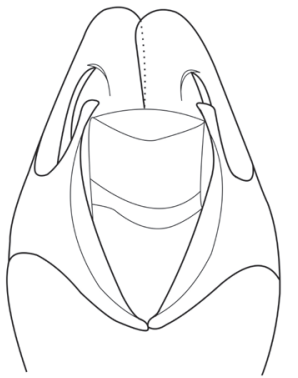

19

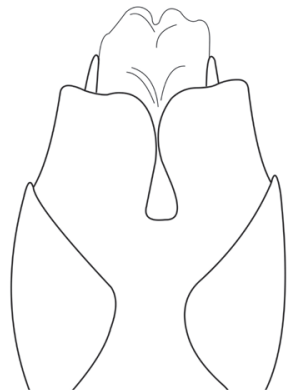

17

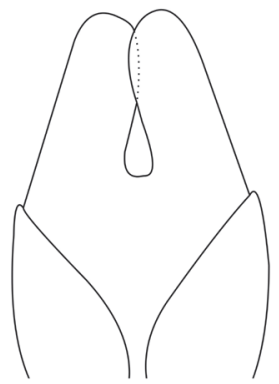

20

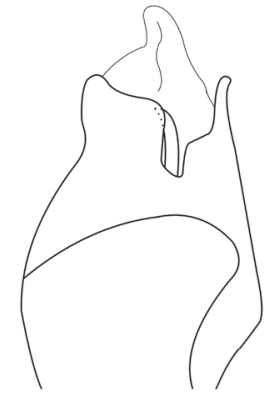

18

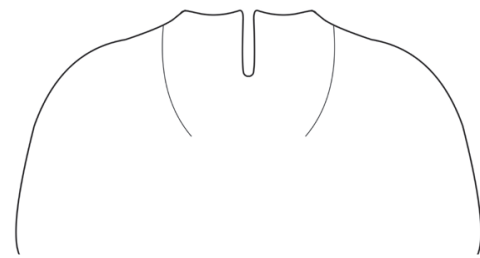

22

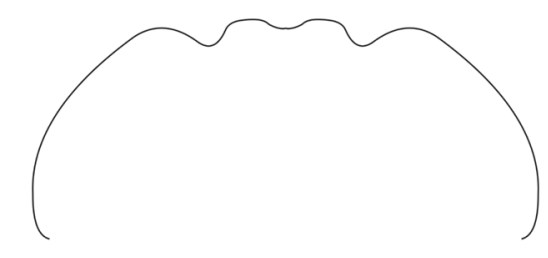

23

Figures 16-23. 16-21. Aedeagus (16, 19 ventral view 17, 20 dorsal view 18, 21 lateral view). 22-23 female abdominal sternite VIII, ventral view 16-18 Themus (Haplothemus) licenti Pic, 1938 19-21, 23 Lycocerus dimidiaticrus (Fairmaire, 1889) 22 Themus (Haplothemus) coriaceipennis (Fairmaire, 1889). Scale bars: $1 \mathrm{~mm}$.

\section{Lycocerus swampingatus (Pic, 1916), comb. n.}

http://species-id.net/wiki/Lycocerus_swampingatus

Figs $19-21$

Cantharis swampingata Pic 1916: 4.

Type material examined. Cantharis swampingata: Holotype $\widehat{\partial}(\mathrm{MNHN})$ : [p]"Swamping \China", [h] "swampingata \Pic", [h]"n. sp.", [h] "Lycocerus \swampingatus (Pic) \det. Y. X. Yang, 2009”, [p] “HOLOTYPUS”. 
Distribution. China (Sichuan).

Remarks. The type specimen of this species was damaged seriously, lacking the abdomen, thorax, all legs, one elytron and part of head, but its aedeagus has been kept well, of which dorsal plates of parameres are separated (Figs 19-21), which is a diagnostic character of Lycocerus (Okushima, 2005). This species is similar to L. canthariformis (Ishida, 1986) (located in Japan) in the pronotum, which is rounded, wider than long, lateral margins are arcuate and posterior angles rounded, but the aedeagus is differs from that of the latter. Also, it is related to L. pubicollis (Heyden, 1889) in the aedeagus, but obviously different from the latter in the pronotum. Consequently, we suggest the following new combination: Lycocerus swampingatus (Pic, 1916) comb. $\mathrm{n}$.

\section{Acknowledgements}

We are grateful to late Dr. Michel Brancucci (NHMB) for his various help in both the study and life when the sensior author visited the Naturhistorisches Museum Basel. Also, we appreciate Dr. Yûichi Okushima (Kurashiki Museum of Natural History, Japan) for his valuable suggestions and sharing some type images in the study, Dr. Vladimír Švihla (NMPC) and Dr. Antoine Mantilleri (MNHN) for providing us a chance to access to the collections under their charge and their generous help to the sensior author when she visited their museums. Thanks are due to Mr. John MacDermott (USA) for correcting our English.

This study is supported by the National Basic Program of China (973 Program) (No. 2011CB302102), the National Natural Science Foundation of China (No. 31010103913, No. 31172135), the Knowledge Innovation of Chinese Academy of Sciences (Nos. KSCX2-EW-G-4, KSCX2-EW-Z-8) and a grant (No. O529YX5105) from the Key Laboratory of the Zoological Systematics and Evolution of the Chinese Academy of Sciences.

\section{References}

Delkeskamp K (1977) Pars 165, Fasc. 1. Editio seconda. Cantharidae. In: Wilcox JA (Ed) Coleopterorum Catalogus Supplementa. W. Junk, The Hague, 1-485.

Fairmaire L (1889) Coléoptères de l'intérieur de la Chine. (5ème partie). Annales de la Société Entomologique de France (6)9: 5-84.

Fairmaire L (1891) Coléoptères de l'intérieur de la Chine. (7ème partie). Bulletin ou Comptes Rendus des Séances de la Société Entomologique de Belgique 35: clxxxvii-ccxxiii.

Gorham HS (1889) Descriptions of new species and a new genus of Coleoptera of the family Telephoridae. Proceedings of the Zoological Society 1889: 96-111.

International Code of Zoological Nomenclature (1999) International code of zoological nomenclature adopted by the International Union of Biological Resources International Commission on Zoological Nomenclature. 4th edn. The International Trust for Zoological Nomenclature, London. 
Jacobson GG (1911) Zhuki Rossii i Zapadnoy Evropy. Rukovodstvo k opredeleniyu zhukov.

Vypusk 9. A. F. Devrjen, St-Pétersburg, 641-720.

Kazantsev SV (2007) Cantharidae. In: Löbl I, Smetana A (Eds) Catalogue of Palaearctic Coleoptera, Vol. 4. Apollo Books, Stenstrup, 47-54.

Kazantsev SV, Brancucci M (2007) Cantharidae. In: Löbl I, Smetana A (Eds) Catalogue of Palaearctic Coleoptera, Vol. 4. Apollo Books, Stenstrup, 234-298.

Kiesenwetter EAH (1874) Die Malacodermen Japans nach dem Ergebnisse der Sammlungen des Herrn G. Lewis während der Jahre 1869-1871. Berliner Entomologische Zeitschrift 18: 241-288. doi: 10.1002/mmnd.18740180302

Okushima Y (2005) A taxonomic study on the genus Lycocerus (Coleoptera, Cantharidae ) from Japan, with zoogeographical considerations. Japanese Journal of Systematic Entomology, Monographic Series 2: 1-383.

Pic M (1906) Noms nouveaux et diagnoses de "Cantharini " (Telephorides) européens et exotiques. L' Échange, Revue Linnéenne 22: 81-85.

Pic M (1916) Diagnoses abrégées diverses. Mélanges Exotico-Entomologiques 21: 2-20.

Pic M (1921) Nouveautés diverses. Mélanges Exotico-Entomologiques 33: 1-32.

Pic M (1922) Nouveautés diverses. Mélanges Exotico-Entomologiques 36: 1-32.

Pic M (1926a) Malacodermes exotiques. L’Échange, Revue Linnéenne 42 [hors-texte] (424-426): 21-36.

Pic M (1926b) Sept coléoptères exotiques nouveaux. Bulletin de la Société Entomologique de France 1926: 153-155.

Pic M (1929) Coléoptères exotiques en partie nouveaux (Suite). L'Échange, Revue Linnéenne 45: 7-8.

Pic M (1933) Schwedisch-chinesische wissenschaftliche Expedition nach den nordwestlichen Provinzien Chinas unter Leitung von Dr. Sven Hedin und Prof. Sü Ping-chang. Insekten gesammelt vom schwedischen Arzt der Expedition Dr. David Hummel 1927-1930. 16. Coleoptera. 2. Helmidae, Dermestidae, Anobiidae, Cleridae, Malacodermata, Dascillidae, Heteromera (ex p.), Bruchidae, Cerambycidae, Phytophaga (ex p.). Arkiv för Zoologi A27(2): 1-14.

Pic M (1938a) Malacodermes exotiques. L’Échange, Revue Linnéenne 54 [hors-texte](472474): 161-164.

Pic M (1938b) Divers coléoptères nouveaux de la Presqu'ile Malaise (IV). Journal of the Federal Malaysian State Museum 18(2): 279-286.

Pic M (1955) Descriptions diverses. Diversités Entomologiques 14: 7-20.

Švihla V (2004) New taxa of the subfamily Cantharinae (Coleoptera, Cantharidae ) from southeastern Asia with notes on other species. Entomologica Basiliensia 26: 155-238.

Švihla V (2005) New taxa of the subfamily Cantharinae (Coleoptera: Cantharidae ) from south-eastern Asia with notes on other species II. Acta Entomologica Musei Nationalis Pragae 45: 71-110.

Švihla V (2008) Redescription of the subgenera of the genus Themus Motschulsky, 1858, with description of five new species (Coleoptera: Cantharidae ). Veröffentlichungen des Naturkundemuseums Erfurt 27: 183-190. 
Wang S-J, Yang J-K (1992) Coleoptera: Cantharidae . In: Huang F-S (Ed) Insects of Wuling mountains area, SW China. Science Press, Beijing, 264-267. [in Chinese]

Wittmer W (1951) Neue Cantharidae aus Herrn Joh. Klapperichs' Südchina Ausbeute (14. Beitrag zur Kenntnis der palaearktischen Malacodermata Col.). Entomologische Blätter für Biologie und Systematik der Käfer 47: 96-103.

Wittmer W (1961) Synonymische und systematische Notizen über Malacodermata (Col.). Entomologischen Arbeiten aus dem Museum G. Frey 12(2): 362-364.

Wittmer W (1969) Synonymische und systematische Notizen über Coleopteren. Mitteilungen der Schweizerischen Entomologischen Gesellschaft 42(1-2): 126-134.

Wittmer W (1972a) Synonymische und systematische Notizen sowie neue Taxa in Cantharidae (Col.). Verhandlungen der Naturforschenden Gesellschaft in Basel 82(1): 105-121.

Wittmer W (1972b) Beitrag zur Kenntnis der palaearktischen Cantharidae und Malachiidae (Col.). Entomologische Arbeiten aus dem Museum G. Frey 23: 122-141.

Wittmer W (1975) 61. Beitrag zur Kenntnis der palaearktischen Cantharidae und Malachiidae. Mitteilungen der Schweizerischen Entomologischen Gesellschaft 48(3-4): 259-267.

Wittmer W (1982) 71. Beitrag zur Kenntnis der palaearktischen Cantharidae. Entomologica Basiliensia 7: 340-347.

Wittmer W (1983a) Beitrag zur einer Revision der Gattung Themus Motsch. Coleoptera: Cantharidae. Entomologischen Arbeiten aus dem Museum G. Frey 31-32: 189-239.

Wittmer W (1983b) Die Familie Cantharidae (Col.) auf Taiwan (2. Teil). Entomological Review of Japan 38(2): 147-172.

Wittmer W (1995) Zur Kenntnis Gattung Athemus Lewis (Col. Cantharidae ). Entomologica Basiliensia 18: 171-286.

Wittmer W (1997) Neue Cantharidae (Col.) aus dem indo-malaiischen und palaearktischen Faunengebiet mit Mutationen. 2. Beitrag. Entomologica Basiliensia 20: 223-366. 